\title{
Delta Shock Wave for the Suliciu Relaxation System
}

\author{
Richard De la cruz, ${ }^{1,2}$ Juan Galvis, ${ }^{2}$ Juan Carlos Juajibioy, ${ }^{2}$ and Leonardo Rendón ${ }^{2}$ \\ ${ }^{1}$ School of Mathematics and Statistics, Universidad Pedagógica y Tecnológica de Colombia, Tunja, Colombia \\ ${ }^{2}$ Department of Mathematics, Universidad Nacional de Colombia, Bogotá, Colombia
}

Correspondence should be addressed to Richard De la cruz; richard.delacruz@uptc.edu.co

Received 13 March 2014; Accepted 27 May 2014; Published 18 June 2014

Academic Editor: Manuel De León

Copyright (C) 2014 Richard De la cruz et al. This is an open access article distributed under the Creative Commons Attribution License, which permits unrestricted use, distribution, and reproduction in any medium, provided the original work is properly cited.

\begin{abstract}
We study the one-dimensional Riemann problem for a hyperbolic system of three conservation laws of Temple class. This system is a simplification of a recently proposed system of five conservations laws by Bouchut and Boyaval that model viscoelastic fluids. An important issue is that the considered $3 \times 3$ system is such that every characteristic field is linearly degenerate. We show an explicit solution for the Cauchy problem with initial data in $L^{\infty}$. We also study the Riemann problem for this system. Under suitable generalized Rankine-Hugoniot relation and entropy condition, both existence and uniqueness of particular delta-shock type solutions are established.
\end{abstract}

\section{Introduction}

The modeling of viscoelastic materials and fluids is important for many applications. In particular, a viscoelastic fluid is a material that exhibits both viscous and elastic characteristics upon deformation. Examples of viscoelastic fluids that are important for applications are latex paint, gelatin, unset cement, liquid acrylic, asphalt, and biological fluids such as synovial fluids, among others. In [1] the authors introduced a new system of conservation laws that models shallow viscoelastic fluids. This new system is motivated by Bouchut and Boyaval in the equation (5.6) of [6]

$$
\begin{gathered}
\rho_{t}+(\rho u)_{x}=0, \\
(\rho u)_{t}+\left(\rho u^{2}+\pi\right)_{x}=0, \\
\left(\rho \frac{\pi}{s^{2}}\right)_{t}+\left(\rho u \frac{\pi}{s^{2}}+u\right)_{x}=0, \\
s_{t}+u s_{x}=0, \\
c_{t}+u c_{x}=0,
\end{gathered}
$$

where $\rho$ denotes the layer depth of fluid, $u$ is the horizontal velocity, $s$ is related to the stress tensor and it is a conserved quantity, $\pi$ is the relaxed pressure, and $c>0$ is introduced in order to parametrize the speeds. This system describes a simple model for a thin layer of non-Newtonian viscoelastic fluid over a given topography at the bottom when the movement is driven by gravitational forces such as geophysical flows (mud flows, landslides, and debris avalanches).

In [2], since $s$ is a conserved quantity, the author considers the case $s=$ const. $>0$. Additionally, we observe that the field $c$ does not appear in the first four equations and, in order to simply notation, introduce the new variable $v=\pi / s^{2}$. After this observation, the following simplified viscoelastic shallow fluid model is obtained:

$$
\begin{gathered}
\rho_{t}+(\rho u)_{x}=0, \\
(\rho u)_{t}+\left(\rho u^{2}+s^{2} v\right)_{x}=0, \\
(\rho v)_{t}+(\rho u v+u)_{x}=0 .
\end{gathered}
$$

We refer to the system above as the Suliciu relaxation system [3-5]. This system can be considered as a relaxation for the isentropic Chaplygin gas dynamics system

$$
\begin{gathered}
\rho_{t}+(\rho u)_{x}=0, \\
(\rho u)_{t}+\left(\rho u^{2}+P\right)_{x}=0,
\end{gathered}
$$


where $\rho$ and $u$, respectively, stand for the density and the velocity of the gas, while the pressure $P$ is given by the state equation $P(\rho)=-s^{2} / \rho$ with $s=$ constant $>0$.

We note that the Suliciu relaxation system (2) is of Temple class and, therefore, it is also of Rich type but it is not diagonal, so its analysis is not standard. We also mention that, when dealing with the system (2), one of the main difficulties is to obtain existence and uniqueness of solutions of Cauchy problems in the presence of vacuum regions, that is, regions where the layer deep $\rho=0$. The existence of global weak solutions, including vacuum regions, was obtained in [2] using the vanishing viscosity method in conjunction with the compensated compactness argument. We also note that there are numerous studies on existence and uniqueness for general Rich type and Temple class system [6-13]. However, some of these results do not apply to (2) since it has all fields being linearly degenerate and the initial data may have oscillations.

The Riemann problem for the Suliciu relaxation system has been extensively studied, for instance in $[3,14]$. We think that there are other cases to investigate. Now, we propose delta wave solutions type for the Suliciu relaxation system.

In this paper we obtain explicit solutions for a Cauchy problem associated with the Suliciu relaxation system (2) with $\rho_{0}(x) \geq \rho>0$, a.e. $x \in \mathbb{R}$, with a possibly oscillating initial data. Also, we construct the Riemann solution for the system focusing our attention on delta shock waves of certain type. The existence and uniqueness of solutions involving delta shock waves can be obtained by solving the generalized Rankine-Hugoniot relation under an entropy condition [15, 16].

The paper is organized as follows. In Section 2 we present the problem and put conditions on the initial data for physical properties are maintained in $\rho$. In Section 3, we show the explicit solutions for the Cauchy problem associated with the Suliciu relaxation system (2) without the presence of vacuum regions. In Section 4, we solve the Riemann problem and we observe that the first and third contact discontinuity are asymptotic to the vacuum. In the last section, we study the existence and uniqueness of solutions delta shock waves type.

\section{Properties of the Suliciu Relaxation System and Some Assumptions}

The eigenvalues associated with the system (2) are given by

$$
\lambda_{1}=u-\frac{s}{\rho}, \quad \lambda_{2}=u, \quad \lambda_{3}=u+\frac{s}{\rho},
$$

where the corresponding Riemann invariants are

$$
R_{1}=s^{2} v-s u, \quad R_{2}=v+\frac{1}{\rho}, \quad R_{3}=s^{2} v+s u .
$$

From the expressions for the eigenvalues and the Riemann invariants we obtain

$$
\begin{gathered}
\lambda_{1}=\frac{R_{3}}{s}-s R_{2}, \quad \lambda_{2}=\frac{1}{2 s}\left(R_{3}-R_{1}\right), \\
\lambda_{3}=s R_{2}-\frac{R_{1}}{s} .
\end{gathered}
$$

From here we can see that system (2) is linearly degenerate. On the other hand, we have that, for each $i, j, k \in\{1,2,3\}$ with $j \neq i, k \neq i$, it holds that

$$
\frac{\partial}{\partial R_{j}}\left(\frac{\left(\partial \lambda_{i} / \partial R_{k}\right)}{\lambda_{k}-\lambda_{i}}\right)=\frac{\partial}{\partial R_{k}}\left(\frac{\left(\partial \lambda_{i} / \partial R_{j}\right)}{\lambda_{j}-\lambda_{i}}\right) .
$$

This means that system (2) is of Rich type. We recall that this classifications is due to [17].

In this paper we focus on the study of the Suliciu relaxation system of conservation laws (2) with bounded initial data

$$
\begin{aligned}
&(\rho(0, x), u(0, x), v(0, x)) \\
&=\left(\rho_{0}(x), u_{0}(x), v_{0}(x)\right), \quad x \in \mathbb{R} \\
& \rho_{0}(x) \geq \underline{\rho}=\text { const. }>0,
\end{aligned}
$$

subject to the following conditions.

(H1) The functions $\rho_{0}, u_{0}$, and $v_{0}$ satisfy

$$
\begin{gathered}
c_{1} \leq u_{0}(x)-s v_{0}(x) \leq c_{2}, \quad c_{3} \leq u_{0}(x)+s v_{0}(x) \leq c_{4}, \\
v_{0}(x)+\frac{1}{\rho_{0}(x)}>c_{5},
\end{gathered}
$$

where $c_{i}, i=1, \ldots, 5$, are suitable constants satisfying

$$
c_{5}-\frac{c_{4}-c_{1}}{2 s}>0
$$

(H2) The total variations of $u_{0}(x)-s v_{0}(x)$ and $u_{0}(x)+$ $s v_{0}(x)$ are bounded.

The conditions ( $\mathrm{H} 1)$ and $(\mathrm{H} 2)$ are somehow natural to impose since they ensure that $\rho$ is positive giving a physical meaning to the Suliciu relaxation system (2).

As we mentioned before, we note that, in [2], the author shows existence of solutions for the Cauchy problem (2)(8) for the case $\rho_{0}(x) \geq 0$. This is done using the vanishing viscosity method and a compensated compactness argument. In [2] it is also shown that all entropies associated with (2) are of the form

$$
\eta(\rho, u, v)=\rho\left(F(u+s v)+G(u-s v)+H\left(v+\frac{1}{\rho}\right)\right),
$$

where $F, G$, and $H$ are arbitrary functions having entropy flux

$$
\begin{aligned}
q(\rho, u, v)= & (\rho u+s) F(u+s v) \\
& +(\rho u-s) G(u-s v)+\rho u H\left(v+\frac{1}{\rho}\right) .
\end{aligned}
$$

Moreover, if the functions $F, G$ and $H$ are convex, then the entropy is also convex (see [2, Theorem 2)]. Thus, from each convex pair $(\eta, q)$ we have the following condition:

$$
\eta_{t}(\rho, u, v)+q_{x}(\rho, u, v)=0
$$

in the sense of distributions. 


\section{Explicit Solutions}

In this section we obtain explicit solutions for the Cauchy problem associated with Suliciu relaxation system with initial data (8) subject to (H1)-(H2) conditions. For this purpose, we used the results given by Wagner, Weinan and Kohn, Li, Peng and Ruiz (for example see [12, 18-20]).

We use the Euler-Lagrange (E-L) transformation $(t, x) \rightarrow$ $(t, y)=(t, Y(t, x))$ defined by

$$
d y=\rho d x-\rho u d t, \quad Y(0, x)=Y_{0}(x) \stackrel{\text { def }}{=} \int_{0}^{x} \rho_{0}(\xi) d \xi .
$$

In Lagrangian coordinates, the system (2) becomes

$$
\begin{aligned}
& \omega_{t}-v_{y}=0, \\
& v_{t}+s^{2} \kappa_{y}=0, \\
& \kappa_{t}+v_{y}=0,
\end{aligned}
$$

where $\omega$ denotes the quantity $1 / \rho$ in Lagrangian coordinates; that is, $\omega(t, y)=(1 /(\rho(t, x)))$, and we also have $\nu(t, y)=$ $u(t, x)$ and $\kappa(t, y)=v(t, x)$.

The eigenvalues associated with (15) are given by

$$
\tilde{\lambda}_{1}=-s, \quad \tilde{\lambda}_{2}=0, \quad \tilde{\lambda}_{3}=s,
$$

and the corresponding Riemann invariants are given by

$$
R_{1}=s^{2} \kappa-s \nu, \quad R_{2}=v+\omega, \quad R_{3}=s^{2} \kappa-s \nu .
$$

In Lagrangian coordinates the entropy condition (13) transforms into

$$
\tilde{\eta}_{t}(\omega, \nu, \kappa)+\tilde{q}_{x}(\omega, \nu, \kappa)=0,
$$

for each $\tilde{\eta}$ with

$$
\begin{gathered}
\tilde{\eta}(\omega, \nu, \kappa)=F(\nu+s \kappa)+G(\nu-s \kappa)+H(\omega+\kappa), \\
\tilde{q}(\omega, \nu, \kappa)=s F(\nu+s \kappa)-s G(\nu-s \kappa),
\end{gathered}
$$

where $F, G$, and $H$ are (arbitrary) convex functions.

The initial condition (8) becomes

$$
\begin{gathered}
(\omega(0, y), v(0, y), \kappa(0, y))=\left(\omega_{0}(y), v_{0}(y), \kappa_{0}(y)\right), \\
y \in \mathbb{R}, \\
\omega_{0}(y) \geq \underline{\omega}>0 .
\end{gathered}
$$

Due to the fact that system (15) is linear, the explicit solution of the corresponding Cauchy problem (15)-(20) is

$$
\begin{aligned}
\omega(t, y)= & \omega_{0}(y)+\kappa_{0}(y)-\kappa(t, y), \\
v(t, y)= & \frac{1}{2}\left(\nu_{0}(y+s t)+\nu_{0}(y-s t)\right) \\
& -\frac{s}{2}\left(\kappa_{0}(y+s t)-\kappa_{0}(y-s t)\right),
\end{aligned}
$$

$$
\begin{aligned}
\kappa(t, y)= & \frac{1}{2}\left(\kappa_{0}(y+s t)+\kappa_{0}(y-s t)\right) \\
& -\frac{1}{2 s}\left(\nu_{0}(y+s t)-\nu_{0}(y-s t)\right) .
\end{aligned}
$$

Moreover, by condition (H1) we obtain that

$$
\begin{gathered}
c_{1} \leq \nu(t, y)-s \kappa(t, y) \leq c_{2}, \quad c_{3} \leq \nu(t, y)+s \kappa(t, y) \leq c_{4}, \\
\omega(t, y)+\kappa(t, y)>c_{5},
\end{gathered}
$$

and since $\rho_{0}(x) \geq \rho=$ const. $>0$ by (8), we have that $\omega(t, y) \geq$ $\underline{\omega}>0$, ensuring that the function $y \mapsto X(t, y)$ is invertible and bi-Lipschitzian from $\mathbb{R}$ to $\mathbb{R}$, for all $t \geq 0$, and by $[12,18]$, we also have uniqueness of the entropy solution of (2)-(8) if and only if we have uniqueness of the entropy solution of (15)-(20).

Therefore, we consider $X_{0}=Y_{0}^{-1}$. Then, the unique function $x=X(t, y)$ that satisfies $X(0, y)=X_{0}(y)$ is given by

$$
\begin{aligned}
X(t, y)= & \frac{1}{2 s} \int_{y-s t}^{y+s t} u_{0}\left(X_{0}(\xi)\right) d \xi \\
& +\int_{0}^{y}\left(v_{0}\left(X_{0}(\xi)\right)+\frac{1}{\rho_{0}\left(X_{0}(\xi)\right)}\right) d \xi \\
& -\frac{1}{2} \int_{0}^{y+s t} v_{0}\left(X_{0}(\xi)\right) d \xi \\
& -\frac{1}{2} \int_{0}^{y-s t} v_{0}\left(X_{0}(\xi)\right) d \xi .
\end{aligned}
$$

Now, we show the result based on the ideas proposed by Weinan and Kohn [19].

Lemma 1. Let $\left(\rho_{1}, u_{1}, v_{1}\right),\left(\rho_{2}, u_{2}, v_{2}\right) \in L^{\infty}\left(\mathbb{R}^{+} \times \mathbb{R}\right)$ be two weak solutions of Suliciu relation system (2). Let $\rho_{0}(x), u_{0}(x), v_{0}(x) \in L^{\infty}(\mathbb{R})$ be such that $\rho_{0}(x) \geq \underline{\rho}>0$, conditions (H1), (H2), and

$$
\inf _{x \in \mathbb{R}}\left(u_{0}(x)+\frac{s}{\rho_{0}(x)}\right)>\sup _{x \in \mathbb{R}}\left(u_{0}(x)-\frac{s}{\rho_{0}(x)}\right),
$$

are satisfied. Suppose that, for any $C^{1}$ function $\phi(x)$ with compact support,

$$
\begin{array}{r}
\lim _{t \rightarrow 0+} \int_{\mathbb{R}} \rho_{i}(t, x) \phi(x) d x=\int_{\mathbb{R}} \rho_{0}(x) \phi(x) d x, \\
\lim _{t \rightarrow 0+} \int_{\mathbb{R}} u_{i}(t, x) \phi(x) d x=\int_{\mathbb{R}} u_{0}(x) \phi(x) d x, \\
\lim _{t \rightarrow 0+} \int_{\mathbb{R}} v_{i}(t, x) \phi(x) d x=\int_{\mathbb{R}} v_{0}(x) \phi(x) d x, \\
\text { for } i=1,2 .
\end{array}
$$

Then $\rho_{1}=\rho_{2}, u_{1}=u_{2}$, and $v_{1}=v_{2}$ almost everywhere. 
Proof. Consider $y_{1}(t, x)$ a solution of

$$
\frac{\partial y_{1}}{\partial x}=\rho_{1}(t, x), \quad \frac{\partial y_{1}}{\partial t}=\rho_{1}(t, x) u_{1}(t, x) .
$$

A solution for (26) exists because the consistency condition is the first equation of (2). Let

$$
y_{1}(0, x)=\int_{0}^{x} \rho_{0}(\xi) d \xi
$$

then $y_{1}$ is unique and by $(\mathrm{H} 1)-(\mathrm{H} 2)$ conditions the map $(t, x) \rightarrow\left(t, y_{1}(t, x)\right)$ is a bi-Lipschitz homeomorphism of $\mathbb{R}^{+} \times \mathbb{R}$ onto itself. Observe that

$$
\begin{gathered}
\omega^{1}\left(t, y_{1}\right)=\frac{1}{\rho_{1}(t, x)}, \quad v^{1}\left(t, y_{1}\right)=u_{1}(t, x), \\
\kappa^{1}\left(t, y_{1}\right)=v_{1}(t, x),
\end{gathered}
$$

is a weak solution of

$$
\begin{gathered}
\omega_{t}^{1}+\kappa_{t}^{1}=0, \\
v_{t}^{1}+s^{2} \kappa_{y_{1}}^{1}=0, \\
\kappa_{t}^{1}+v_{y_{1}}^{1}=0 .
\end{gathered}
$$

Let $\psi(y)$ be a continuous function with compact support. Then

$$
\begin{aligned}
\int \psi(y) \omega^{1}(t, y) d y & =\int \psi\left(y_{1}(t, x)\right) \omega^{1}\left(t, y_{1}(t, x)\right) \frac{\partial y_{1}}{\partial x} d x \\
& =\int \psi\left(y_{1}(t, x)\right) d x,
\end{aligned}
$$

thereby

$$
\lim _{t \rightarrow 0+} \int \psi(y) \omega^{1}(t, y) d y=\int \psi\left(y_{1}(0, x)\right) d x .
$$

Similarly,

$$
\begin{aligned}
& \lim _{t \rightarrow 0+} \int \psi(y) \nu^{1}(t, y) d y=\int \psi\left(y_{1}(0, x)\right) \rho_{0}(x) u_{0}(x) d x, \\
& \lim _{t \rightarrow 0+} \int \psi(y) \kappa^{1}(t, y) d y=\int \psi\left(y_{1}(0, x)\right) \rho_{0}(x) v_{0}(x) d x .
\end{aligned}
$$

Now, we can do a transformation for $\left(\rho_{2}, u_{2}, v_{2}\right)$. If $y_{2}(t, x)$ solve

$$
\begin{gathered}
\frac{\partial y_{2}}{\partial x}=\rho_{2}(t, x), \quad \frac{\partial y_{2}}{\partial t}=\rho_{2}(t, x) u_{2}(t, x), \\
y_{2}(0, x)=\int_{0}^{x} \rho_{0}(\xi) d \xi,
\end{gathered}
$$

and choosing

$$
\begin{gathered}
\omega^{2}\left(t, y_{2}\right)=\frac{1}{\rho_{2}(t, x)}, \quad v^{2}\left(t, y_{2}\right)=u_{2}(t, x), \\
\kappa^{2}\left(t, y_{2}\right)=v_{2}(t, x) .
\end{gathered}
$$

Also $\left(\omega^{2}, v^{2}, \kappa^{2}\right)$ is a weak solution of

$$
\begin{gathered}
\omega_{t}^{2}+\kappa_{t}^{2}=0, \\
v_{t}^{2}+s^{2} \kappa_{y_{2}}^{2}=0, \\
\kappa_{t}^{2}+v_{y_{2}}^{2}=0,
\end{gathered}
$$

with

$$
\begin{gathered}
\lim _{t \rightarrow 0+} \int \psi(y) \omega^{2}(t, y) d y=\int \psi\left(y_{2}(0, x)\right) d x, \\
\lim _{t \rightarrow 0+} \int \psi(y) \nu^{2}(t, y) d y=\int \psi\left(y_{2}(0, x)\right) \rho_{0}(x) u_{0}(x) d x, \\
\lim _{t \rightarrow 0+} \int \psi(y) \kappa^{2}(t, y) d y=\int \psi\left(y_{2}(0, x)\right) \rho_{0}(x) v_{0}(x) d x .
\end{gathered}
$$

Since $\left(\rho_{1}, u_{1}, v_{1}\right)$ and $\left(\rho_{2}, u_{2}, v_{2}\right)$ have the same initial data, $y_{1}(0, x)=y_{2}(0, x)$ and from the dummy variables $y_{1}$ and $y_{2}$, $\left(\omega^{1}, \nu^{1}, \kappa^{1}\right)$ and $\left(\omega^{2}, v^{2}, \kappa^{2}\right)$ solve the same linear hyperbolic equation with the same initial condition. Therefore, $\omega^{1}(t, y)=$ $\omega^{2}(t, y), \nu^{1}(t, y)=\nu^{2}(t, y)$, and $\kappa^{1}(t, y)=\kappa^{2}(t, y)$ for all $(t, y) \in \mathbb{R}^{+} \times \mathbb{R}$.

To conclude that $\rho_{1}=\rho_{2}, u_{1}=u_{2}$, and $v_{1}=v_{2}$ we must show that $y_{1}(t, x)=y_{2}(t, x)$.

The Jacobian of the map $\left(t, y_{1}\right) \rightarrow(t, x)$ is the inverse of that of the map $(t, x) \rightarrow\left(t, y_{1}\right)$, so

$$
\begin{gathered}
\frac{\partial x}{\partial y_{1}}=\omega^{1}\left(t, y_{1}\right), \quad \frac{\partial x}{\partial t}=v^{1}\left(t, y_{1}\right), \\
x\left(0, y_{1}\right)=\left[y_{1}(0, x)\right]^{-1} .
\end{gathered}
$$

A similar relation holds for $y_{2}$. Since $\omega^{1}=\omega^{2}, v^{1}=v^{2}$, and $\kappa^{1}=\kappa^{2}$, we see that $x\left(t, y_{1}\right)=x\left(t, y_{2}\right)$, and it follows that $y_{l}=y_{2}$.

From the above, we obtain the following theorem.

Theorem 2. Assume that $\rho_{0}, u_{0}, v_{0} \in L^{\infty}(\mathbb{R})$ with $\rho_{0}(x) \geq \rho>$ 0, a.e. $x \in \mathbb{R}$, the conditions (H1), (H2) hold and

$$
\inf _{x \in \mathbb{R}}\left(u_{0}(x)+\frac{s}{\rho_{0}(x)}\right)>\sup _{x \in \mathbb{R}}\left(u_{0}(x)-\frac{s}{\rho_{0}(x)}\right) .
$$

Then, the Cauchy problems (2)-(8) has a unique global solution $(\rho, u, v) \in L^{\infty}\left(\mathbb{R}^{+} \times \mathbb{R}\right)$ that satisfies the entropy condition (13) for all pair $(\eta, q)$ defined in (11)-(12). Moreover, this solution is given by

$$
\begin{gathered}
\rho(t, x) \\
=\frac{\rho_{0}\left(X_{0}(Y(t, x))\right)}{1+\rho_{0}\left(X_{0}(Y(t, x))\right)\left[v_{0}\left(X_{0}(Y(t, x))\right)-v(t, x)\right]}, \\
u(t, x)=\Gamma_{u_{0}}^{+}(t, x)-s \Gamma_{v_{0}}^{-}(t, x), \\
v(t, x)=\Gamma_{v_{0}}^{+}(t, x)-\frac{1}{s} \Gamma_{u_{0}}^{-}(t, x),
\end{gathered}
$$


where

$$
\begin{aligned}
& \Gamma_{g_{0}}^{ \pm}(t, x) \\
& \quad=\frac{1}{2}\left[g_{0}\left(X_{0}(Y(t, x)+s t)\right) \pm g_{0}\left(X_{0}(Y(t, x)-s t)\right)\right]
\end{aligned}
$$

Now we apply our result to particular example, which behaves as the advection equation.

Example 3. We consider the initial data $u_{0}(x)=\bar{u}$ and $v_{0}(x)=\bar{v}$ as being constant functions, and $\rho_{0}(x) \geq 0$, a.e. $x \in \mathbb{R}$, as a bounded function.

By the E-L transformation,

$$
Y_{0}(x)=\int_{0}^{x} \rho_{0}(\xi) d \xi, \quad \text { consider } X_{0}=Y_{0}^{-1}
$$

Then,

$$
X(t, y)=X_{0}(y)+\bar{u} t, \quad Y(t, x)=Y_{0}(x-\bar{u} t) .
$$

In this way the solution of the Cauchy problem is given by

$$
\rho(t, x)=\rho_{0}(x-\bar{u} t), \quad u(t, x)=\bar{u}, \quad v(t, x)=\bar{v} .
$$

\section{Riemann Problem}

In this section we study the solution for the Riemann problem associated with the Suliciu relaxation system, in which the left and right constant states $\left(\rho_{l}, u_{l}, v_{l}\right)$ and $\left(\rho_{r}, u_{r}, v_{r}\right)$, respectively, satisfy the conditions (H1)-(H2) and $\lambda_{1}\left(\rho_{l}, u_{l}, v_{l}\right)<$ $\lambda_{3}\left(\rho_{r}, u_{r}, v_{r}\right)$

Consider the Riemann problem of the system (2) with initial data

$$
(\rho, u, v)(0, x)= \begin{cases}\left(\rho_{r}, u_{r}, v_{r}\right), & \text { if } x>0, \\ \left(\rho_{l}, u_{l}, v_{l}\right), & \text { if } x<0,\end{cases}
$$

where $\left(\rho_{0}, u_{0}, v_{0}\right)(x)=(\rho, u, v)(0, x)$ satisfies the conditions (H1) and (H2).

First, observe that system (2) is equivalent to

$$
\begin{gathered}
\rho_{t}+m_{x}=0 \\
m_{t}+\left(\frac{m^{2}}{\rho}+s^{2} \frac{n}{\rho}\right)_{x}=0 \\
n_{t}+\left(\frac{m n}{\rho}+\frac{m}{\rho}\right)_{x}=0
\end{gathered}
$$

with $s=$ const. $>0$, where $m=\rho u, n=\rho v$, and the initial data (44) are given by

$$
(\rho, m, n)(0, x)= \begin{cases}\left(\rho_{r}, m_{r}, n_{r}\right), & \text { if } x>0, \\ \left(\rho_{l}, m_{l}, n_{l}\right), & \text { if } x<0\end{cases}
$$

with $m_{r}=\rho_{r} u_{r}, n_{r}=\rho_{r} v_{r}, m_{l}=\rho_{l} u_{l}$, and $n_{l}=\rho_{l} v_{l}$.
The eigenvalues of the system (2), in the variables $\rho, m, n$, are given by

$$
\lambda_{1}=\frac{m-s}{\rho}, \quad \lambda_{2}=\frac{m}{\rho}, \quad \lambda_{3}=\frac{m+s}{\rho},
$$

and the right eigenvectors become

$$
\begin{gathered}
r_{1}(\rho, m, n)=\frac{(\rho, m-s, n+1)}{\sqrt{\rho^{2}+(m-s)^{2}+(n+1)^{2}}}, \\
r_{2}(\rho, m, n)=\frac{(\rho, m, n)}{\sqrt{\rho^{2}+m^{2}+n^{2}}}, \\
r_{3}(\rho, m, n)=\frac{(\rho, m+s, n+1)}{\sqrt{\rho^{2}+(m+s)^{2}+(n+1)^{2}}} .
\end{gathered}
$$

From (48) we get that the 1-rarefaction curve can be found as

$$
\frac{d \rho}{d t}=\frac{\rho}{\sqrt{\rho^{2}+(m-s)^{2}+(n+1)^{2}}}, \quad \rho(0)=\rho_{0},
$$

$$
\begin{array}{ll}
\frac{d m}{d t}=\frac{m-s}{\sqrt{\rho^{2}+(m-s)^{2}+(n+1)^{2}}}, & m(0)=m_{0}, \\
\frac{d n}{d t}=\frac{n+1}{\sqrt{\rho^{2}+(m-s)^{2}+(n+1)^{2}}}, & n(0)=n_{0} .
\end{array}
$$

That is,

$$
\sqrt{\rho^{2}+(m-s)^{2}+(n+1)^{2}}-\sqrt{\rho_{0}^{2}+\left(m_{0}-s\right)^{2}+\left(n_{0}+1\right)^{2}}=t .
$$

Therefore, the integral curves of the vector field $r_{1}$ are given by straight lines in the direction of the vector $r_{1}\left(\rho_{0}, m_{0}, n_{0}\right)$ and goes through the point $(0, s,-1)$; that is,

$$
\begin{aligned}
& \rho=\rho_{0}+\frac{\rho_{0}}{\sqrt{\rho_{0}^{2}+\left(m_{0}-s\right)^{2}+\left(n_{0}+1\right)^{2}}} t, \\
& m=m_{0}+\frac{m_{0}-s}{\sqrt{\rho_{0}^{2}+\left(m_{0}-s\right)^{2}+\left(n_{0}+1\right)^{2}}} t, \quad t \in \mathbb{R} . \\
& m=m_{0}+\frac{n_{0}+1}{\sqrt{\rho_{0}^{2}+\left(m_{0}-s\right)^{2}+\left(n_{0}+1\right)^{2}}} t .
\end{aligned}
$$

Analogously, from (49) we can analyze the 2-rarefaction curve. In this case the integral curves corresponding to the vector field $r_{2}$ are given by straight lines going through the origin in the direction of the vector $r_{2}\left(\rho_{0}, m_{0}, n_{0}\right)$. Also, from (50) we can see that, for the 3-rarefaction curve, the integral curves of the vector field $r_{3}$, are given by straight lines trough $(0,-s,-1)$ that are parallel to $r_{3}\left(\rho_{0}, m_{0}, n_{0}\right)$.

Thus, the $i$-rarefaction curve $R_{i}(\sigma)(\rho, m, n)$ satisfies

$$
R_{i}(\sigma)(\rho, m, n)=(\rho, m, n)+\sigma r_{i}(\rho, m, n) \quad \text { for } i=1,2,3
$$


This also may be deduced from self-similar solution

$$
(\rho, u, v)(t, x)=(\rho, u, v)(\xi), \quad \xi=\frac{x}{t},
$$

for which system (2) becomes

$$
\begin{gathered}
-\xi \rho_{\xi}+(\rho u)_{\xi}=0, \\
-\xi(\rho u)_{\xi}+\left(\rho u^{2}+s^{2} v\right)_{\xi}=0, \\
-\xi(\rho v)_{\xi}+(\rho u v+u)_{\xi}=0,
\end{gathered}
$$

and initial data (44) changes to the boundary condition

$$
\begin{aligned}
& (\rho, u, v)(-\infty)=\left(\rho_{l}, u_{l}, v_{l}\right), \\
& (\rho, u, v)(+\infty)=\left(\rho_{r}, u_{r}, v_{r}\right) .
\end{aligned}
$$

This is a two-point boundary value problem of first-order ordinary differential equations with the boundary values in the infinity.

For smooth solution, (56) is reduced to

$$
\left(\begin{array}{ccc}
u-\xi & \rho & 0 \\
0 & \rho(u-\xi) & 0 \\
0 & 1 & \rho(u-\xi)
\end{array}\right)\left(\begin{array}{l}
\rho \\
u \\
v
\end{array}\right)_{\xi}=0 .
$$

It provides either the general solutions (constant states)

$$
(\rho, u, v)=\text { constant } \quad(\rho>0),
$$

or singular solutions

$$
\begin{array}{rrr}
\xi=\lambda_{1}=u-\frac{s}{\rho}, & d\left(u-\frac{s}{\rho}\right)=0, & d\left(v+\frac{1}{\rho}\right)=0, \\
\xi=\lambda_{2}=u, & d u=0, \quad d v=0, \\
\xi=\lambda_{3}=u+\frac{s}{\rho}, & d\left(u+\frac{s}{\rho}\right)=0, & d\left(v+\frac{1}{\rho}\right)=0 .
\end{array}
$$

Integrating (60) from $\left(\rho_{l}, u_{l}, v_{l}\right)$ to $(\rho, u, v)$, one can get that

$$
\begin{aligned}
& \xi=\lambda_{1}=u-\frac{s}{\rho}, \quad u-\frac{s}{\rho}=u_{l}-\frac{s}{\rho_{l}}, \quad v+\frac{1}{\rho}=v_{l}+\frac{1}{\rho_{l}}, \\
& \xi=\lambda_{2}=u, \quad u=u_{l} \quad v=v_{l}, \\
& \xi=\lambda_{3}=u+\frac{s}{\rho}, \quad u+\frac{s}{\rho}=u_{l}+\frac{s}{\rho_{l}}, \quad v+\frac{1}{\rho}=v_{l}+\frac{1}{\rho_{l}} .
\end{aligned}
$$

Observe that (61) in the variables $\rho, m, n$ is equivalent to (54).

For a bounded discontinuity at $\xi=\omega$, the RankineHugoniot conditions hold. That is,

$$
\begin{gathered}
-\omega[\rho]+[\rho u]=0, \\
-\omega[\rho u]+\left[\rho u^{2}+s^{2} v\right]=0, \\
-\omega[\rho v]+[\rho u v+u]=0,
\end{gathered}
$$

where $[q]=q_{l}-q$ is the jump of $q$ across the discontinuous line and $\omega$ is the velocity of the discontinuity. From (62), we have

$$
\begin{aligned}
& \omega=u-\frac{s}{\rho}, \quad u-\frac{s}{\rho}=u_{l}-\frac{s}{\rho_{l}}, \quad v+\frac{1}{\rho}=v_{l}+\frac{1}{\rho_{l}}, \\
& \omega=u, \quad u=u_{l}, \quad v=v_{l}, \\
& \omega=u+\frac{s}{\rho}, \quad u+\frac{s}{\rho}=u_{l}+\frac{s}{\rho_{l}}, \quad v+\frac{1}{\rho}=v_{l}+\frac{1}{\rho_{l}} \text {. }
\end{aligned}
$$

From (61) and (63), we conclude that the rarefaction waves and the shock waves are coincident, which correspond to contact discontinuities. Namely, for a given left state $\left(\rho_{l}, u_{l}, v_{l}\right)$, the contact discontinuity curves, which are the sets of states that can be connected on the right by a 1-contact discontinuity $J_{1}$, a 2 -contact discontinuity $J_{2}$, or a 3-contact discontinuity $J_{3}$, are as follows:

$$
\begin{gathered}
J_{1}:(\rho, u, v):=\left(\rho, u_{l}-\frac{s}{\rho_{l}}+\frac{s}{\rho}, v_{l}+\frac{1}{\rho_{l}}-\frac{1}{\rho}\right), \\
J_{2}:(\rho, u, v):=\left(\rho, u_{l}, v_{l}\right), \\
J_{3}:(\rho, u, v):=\left(\rho, u_{l}+\frac{s}{\rho_{l}}-\frac{s}{\rho}, v_{l}+\frac{1}{\rho_{l}}-\frac{1}{\rho}\right), \quad \rho>0 .
\end{gathered}
$$

In the space $(\rho>0, u \in \mathbb{R}, u \in \mathbb{R})$, through the point $\left(\rho_{l}, u_{l}, v_{l}\right)$, we draw curves (64) which are denoted by $J_{1}, J_{2}$, and $J_{3}$, respectively. So, $J_{1}$ has asymptotes $\rho=0$ and $\left(\rho, u_{l}-\right.$ $\left.s / \rho_{l}, v_{l}+1 / \rho_{l}\right)$ for $\rho \geq 0$, and $J_{3}$ has asymptotes $\rho=0$ and $\left(\rho, u_{l}+s / \rho_{l}, v_{l}+1 / \rho_{l}\right)$.

In order to solve the Riemann problem (2)-(44), we consider constant left and right states $U_{l}=\left(\rho_{l}, u_{l}, v_{l}\right)$ and $U_{r}=\left(\rho_{r}, u_{r}, v_{r}\right)$, respectively, such that the conditions (H1)-(H2) are satisfied and $\lambda_{1}\left(U_{l}\right)<\lambda_{3}\left(U_{r}\right)$. Then there exists intermediate states, $U_{*}=\left(\rho_{*}, u_{*}, v_{*}\right)$ and $U_{* *}=$ $\left(\rho_{* *}, u_{* *}, v_{* *}\right)$, such that $U_{*}=R_{1}\left(\sigma_{1}\right)\left(U_{l}\right), U_{* *}=R_{2}\left(\sigma_{2}\right)\left(U_{*}\right)$, and $U_{r}=R_{3}\left(\sigma_{3}\right)\left(U_{* *}\right)$, for some $\sigma_{1}, \sigma_{2}$, and $\sigma_{3}$.

Furthermore, because of (54), the states $U^{*}$ and $U^{* *}$ should satisfy

$$
\begin{gathered}
u_{*}=\left(u_{l}-\frac{s}{\rho_{l}}\right)+\frac{s}{\rho_{*}}, \\
v_{*}=\left(v_{l}+\frac{1}{\rho_{l}}\right)-\frac{1}{\rho_{*}}, \\
u_{*}=u_{* *}, \\
v_{*}=v_{* *}, \\
u_{* *}=\left(u_{r}+\frac{s}{\rho_{r}}\right)-\frac{s}{\rho_{* *}}, \\
v_{* *}=\left(v_{r}+\frac{1}{\rho_{r}}\right)-\frac{1}{\rho_{* *}} .
\end{gathered}
$$


From (65d), (65b), and (65f), we have

$$
\frac{1}{\rho_{* *}}-\frac{1}{\rho_{*}}=\left(v_{r}+\frac{1}{\rho_{r}}\right)-\left(v_{l}+\frac{1}{\rho_{l}}\right) .
$$

On the other hand, from (65c), (65a), and (65e), we have

$$
\frac{1}{\rho_{* *}}+\frac{1}{\rho_{*}}=\frac{1}{s}\left\{\left(u_{r}+\frac{s}{\rho_{r}}\right)-\left(u_{l}-\frac{s}{\rho_{l}}\right)\right\} .
$$

From (66) and (67) we conclude that

$$
\begin{aligned}
& \frac{1}{\rho_{*}}=\frac{1}{2 s}\left(\lambda_{3}\left(U_{r}\right)-\lambda_{1}\left(U_{l}\right)\right)-\frac{1}{2}\left(R_{2}\left(U_{r}\right)-R_{2}\left(U_{l}\right)\right), \\
& \frac{1}{\rho^{* *}}=\frac{1}{2 s}\left(\lambda_{3}\left(U_{r}\right)-\lambda_{1}\left(U_{l}\right)\right)+\frac{1}{2}\left(R_{2}\left(U_{r}\right)-R_{2}\left(U_{l}\right)\right) .
\end{aligned}
$$

Thus, (65a), (65b), (65c), (65d), (65c), (65e), (65f), and (68) give

$$
\begin{aligned}
& u_{*}=\frac{1}{2}\left\{\left(u_{l}+s v_{l}\right)+\left(u_{r}-s v_{r}\right)\right\}=u_{* *}, \\
& v_{*}=\frac{1}{2 s}\left\{\left(u_{l}+s v_{l}\right)-\left(u_{r}-s v_{r}\right)\right\}=v_{* *} .
\end{aligned}
$$

Observe that, by conditions (H1) and (H2) on $U_{l}$ and $U_{r}$, we have that $U_{*}$ and $U_{* *}$ also satisfy (H1) and (H2). This guarantees that $\rho_{*}$ and $\rho_{* *}$ are positive.

Note that $\lambda_{1}\left(U^{-}\right)<\lambda_{3}\left(U^{+}\right)$implies

$$
\left|R_{2}\left(U^{+}\right)-R_{2}\left(U^{-}\right)\right|<\frac{1}{s}\left(\lambda_{3}\left(U^{+}\right)-\lambda_{1}\left(U^{-}\right)\right) .
$$

Remark 4. Observe that if $\lambda_{1}\left(U_{l}\right)=\lambda_{3}\left(U_{r}\right)$, then

$$
s=\frac{\left(m_{l} / \rho_{l}\right)-\left(m_{r} / \rho_{r}\right)}{\left(1 / \rho_{r}\right)+\left(1 / \rho_{l}\right)} \text {. }
$$

If we assume that $\rho_{r}=\rho_{l}$ and $n_{r}=n_{l}$, then (71) reduces to $s=\left(\left(m_{l}-m_{r}\right) / 2\right)$. In this case the Riemann problem does not have a solution since the lines $R_{1}(\sigma)\left(U_{l}\right)$ and $R_{3}(\sigma)\left(U_{* *}\right)$ are parallel.

Thus, by conditions (H1), (H2), and $\lambda_{3}\left(U_{r}\right)>\lambda_{1}\left(U_{l}\right)$, the solution of the Riemann problem is given by

$$
\begin{aligned}
& (\rho, u, v)(t, x) \\
& = \begin{cases}\left(\rho_{l}, u_{l}, v_{l}\right), & \text { if } \frac{x}{t}<\lambda_{1}\left(U_{l}\right), \\
\left(\rho_{*}, u_{*}, v_{*}\right), & \text { if } \lambda_{1}\left(U_{l}\right) \leq \frac{x}{t}<\lambda_{2}\left(U_{*}\right), \\
\left(\rho_{* *}, u_{* *}, v_{* *}\right), & \text { if } \lambda_{2}\left(U_{* *}\right) \leq \frac{x}{t}<\lambda_{3}\left(U_{r}\right), \\
\left(\rho_{r}, u_{r}, v_{r}\right), & \text { if } \frac{x}{t} \geq \lambda_{3}\left(U_{r}\right) .\end{cases}
\end{aligned}
$$

Additionally, as usual, since the system is linearly degenerate, $\lambda_{1}\left(U_{l}\right)=\lambda_{1}\left(U_{*}\right), \lambda_{2}\left(U_{*}\right)=\lambda_{2}\left(U_{* *}\right)$, and $\lambda_{3}\left(U_{* *}\right)=\lambda_{3}\left(U_{r}\right)$.
Lemma 5. Given left and right constant states $\left(\rho_{l}, u_{l}, v_{l}\right)$ and $\left(\rho_{r}, u_{r}, v_{r}\right)$, respectively, such that they satisfy conditions (H1), (H2), and $\lambda_{1}\left(\rho_{l}, u_{l}, v_{l}\right)<\lambda_{3}\left(\rho_{r}, u_{r}, v_{r}\right)$ then, the following relation is satisfied

$$
\begin{aligned}
& \left|R_{2}\left(\rho_{r}, u_{r}, v_{r}\right)-R_{2}\left(\rho_{l}, u_{l}, v_{l}\right)\right| \\
& \quad<\frac{1}{s}\left(\lambda_{3}\left(\rho_{r}, u_{r}, v_{r}\right)-\lambda_{1}\left(\rho_{l}, u_{l}, v_{l}\right)\right) .
\end{aligned}
$$

The results of this section can be summarized in the following theorem.

Theorem 6. Given left and right constant states $\left(\rho_{l}, u_{l}, v_{l}\right)$ and $\left(\rho_{r}, u_{r}, v_{r}\right)$, respectively, such that they satisfy conditions (H1), (H2), and $\lambda_{1}\left(\rho_{l}, u_{l}, v_{l}\right)<\lambda_{3}\left(\rho_{r}, u_{r}, v_{r}\right)$, then, there is a unique global solution to the Riemann problem (2)-(44). Moreover, this solution is given by

$$
\begin{aligned}
& (\rho, u, v)(t, x) \\
& =\left\{\begin{array}{l}
\left(\rho_{l}, u_{l}, v_{l}\right), \\
\quad \text { if } x<\lambda_{1}\left(\rho_{l}, u_{l}, v_{l}\right) t, \\
\left(\rho_{*}, u_{*}, v_{*}\right), \\
\quad \text { if } \lambda_{1}\left(\rho_{l}, u_{l}, v_{l}\right) t<x<\lambda_{2}\left(\rho_{* *}, u_{* *}, v_{* *}\right) t, \\
\left(\rho_{* *}, u_{* *}, v_{* *}\right), \\
\quad \text { if } \lambda_{2}\left(\rho_{* *}, u_{* *}, v_{* *}\right) t<x<\lambda_{3}\left(\rho_{r}, u_{r}, v_{r}\right) t, \\
\left(\rho_{r}, u_{r}, v_{r}\right), \\
\quad \text { if } x>\lambda_{3}\left(\rho_{r}, u_{r}, v_{r}\right) t,
\end{array}\right.
\end{aligned}
$$

where

$$
\begin{gathered}
\frac{1}{\rho_{*}}=\frac{1}{2 s}\left(u_{r}-u_{l}\right)-\frac{1}{2}\left(v_{r}-v_{l}\right)+\frac{1}{\rho_{l}}, \\
\frac{1}{\rho_{* *}}=\frac{1}{2 s}\left(u_{r}-u_{l}\right)+\frac{1}{2}\left(v_{r}-v_{l}\right)+\frac{1}{\rho_{r}}, \\
u_{*}=\frac{1}{2}\left\{\left(u_{l}+s v_{l}\right)+\left(u_{r}-s v_{r}\right)\right\}=u_{* *}, \\
v_{*}=\frac{1}{2 s}\left\{\left(u_{l}+s v_{l}\right)-\left(u_{r}-s v_{r}\right)\right\}=v_{* *} .
\end{gathered}
$$

\section{Delta Shock Solution}

In this section, we discuss the solution for the Riemann problem associated with the Suliciu relaxation system, in which the left and right constant states $\left(\rho_{l}, u_{l}, v_{l}\right)$ and $\left(\rho_{r}, u_{r}, v_{r}\right)$, respectively, satisfy the conditions $(\mathrm{H} 1)$ and $(\mathrm{H} 2)$, but unlike previous section they satisfy $\lambda_{1}\left(\rho_{l}, u_{l}, v_{l}\right) \geq \lambda_{3}\left(\rho_{r}, u_{r}, v_{r}\right)$.

Denote by $\operatorname{BM}(\mathbb{R})$ the space of bounded Borel measures on $\mathbb{R}$, and then the definition of a measure solution of Suliciu relaxation system in $\mathrm{BM}(\mathbb{R})$ can be given as follows.

Definition 7. A triple $(\rho, u, v)$ constitutes a measure solution to the Suliciu relaxation system, if it holds that

(a) $\rho \in L^{\infty}((0, \infty), \operatorname{BM}(\mathbb{R})) \cap C\left((0, \infty), H^{-s}(\mathbb{R})\right)$,

(b) $u \in L^{\infty}\left((0, \infty), L^{\infty}(\mathbb{R})\right) \cap C\left((0, \infty), H^{-s}(\mathbb{R})\right)$, 
(c) $v \in L_{\mathrm{loc}}^{\infty}\left((0, \infty), L_{\mathrm{loc}}^{\infty}(\mathbb{R})\right) \cap C\left((0, \infty), H^{-s}(\mathbb{R})\right), s>0$,

(d) $u$ and $v$ are measurable with respect to $\rho$ at almost for all $t \in(0, \infty)$,

and

$$
\begin{gathered}
I_{1}=\int_{0}^{\infty} \int_{\mathbb{R}}\left(\phi_{t}+u \phi_{x}\right) d \rho d t=0, \\
I_{2}=\int_{0}^{\infty} \int_{\mathbb{R}} u\left(\phi_{t}+u \phi_{x}\right) d \rho d t+\int_{0}^{\infty} \int_{\mathbb{R}} s^{2} v \phi_{x} d x d t=0, \\
I_{3}=\int_{0}^{\infty} \int_{\mathbb{R}} v\left(\phi_{t}+u \phi_{x}\right) d \rho d t+\int_{0}^{\infty} \int_{\mathbb{R}} u \phi_{x} d x d t=0,
\end{gathered}
$$

for all test function $\phi \in C_{0}^{\infty}\left(\mathbb{R}^{+} \times \mathbb{R}\right)$.

Definition 8. A two-dimensional weighted delta function $w(s) \delta_{L}$ supported on a smooth curve $L$ parameterized as $t=$ $t(s), x=x(s)(c \leq s \leq d)$ is defined by

$$
\left\langle w(s) \delta_{L}, \phi(t, x)\right\rangle=\int_{c}^{d} w(s) \phi(t(s), x(s)) d s,
$$

for all $\phi \in C_{0}^{\infty}\left(\mathbb{R}^{2}\right)$.

Definition 9. A triple distribution $(\rho, u, v)$ is called a delta shock wave, if it is represented in the form

$$
\begin{aligned}
& (\rho, u, v)(t, x) \\
& \quad= \begin{cases}\left(\rho_{l}, u_{l}, v_{l}\right)(t, x), & x<x(t), \\
\left(w(t) \delta(x-x(t)), u_{\delta}(t), g(t)\right), & x=x(t), \\
\left(\rho_{r}, u_{r}, v_{r}\right)(t, x), & x>x(t),\end{cases}
\end{aligned}
$$

and satisfies Definition 7, where $\left(\rho_{l}, u_{l}, v_{l}\right)(t, x)$ and $\left(\rho_{r}, u_{r}, v_{r}\right)(t, x)$ are piecewise smooth bounded solutions of the Suliciu relaxation system (2).

We set $d x / d t=u_{\delta}(t)$ since the concentration in $\rho$ needs to travel at the speed of discontinuity. Hence, we say that a delta shock wave (78) is a measure solution to the Suliciu relaxation system (2) if and only if the following relation holds:

$$
\begin{gathered}
\frac{d x(t)}{d t}=u_{\delta}(t), \\
\frac{d w(t)}{d t}=-[\rho] u_{\delta}(t)+[\rho u], \\
\frac{d w(t) u_{\delta}(t)}{d t}=-[\rho u] u_{\delta}(t)+\left[\rho u^{2}+s^{2} v\right], \\
\frac{d w(t) g(t)}{d t}=-[\rho v] u_{\delta}(t)+[\rho u v+u] .
\end{gathered}
$$

In fact, for any test function $\phi \in C_{0}^{\infty}\left(\mathbb{R}^{+} \times \mathbb{R}\right)$, from (76), we obtain

$$
\begin{aligned}
I_{1} & =\int_{0}^{\infty} \int_{\mathbb{R}}\left(\phi_{t}+u \phi_{x}\right) d \rho d t \\
& =\int_{0}^{\infty}\left\{-u_{\delta}(t)[\rho]+[\rho u]-\frac{d w(t)}{d t}\right\} \phi d t,
\end{aligned}
$$

$$
\begin{aligned}
I_{2} & =\int_{0}^{\infty} \int_{\mathbb{R}} u\left(\phi_{t}+u \phi_{x}\right) d \rho d t+\int_{0}^{\infty} \int_{\mathbb{R}} s^{2} v \phi_{x} d x d t \\
& =\int_{0}^{\infty}\left\{-u_{\delta}(t)[\rho u]+\left[\rho u^{2}+s^{2} v\right]-\frac{d w(t) u_{\delta}(t)}{d t}\right\} d t \\
I_{3} & =\int_{0}^{\infty} \int_{\mathbb{R}} v\left(\phi_{t}+u \phi_{x}\right) d \rho d t+\int_{0}^{\infty} \int_{\mathbb{R}} u \phi_{x} d x d t \\
& =\int_{0}^{\infty}\left\{-u_{\delta}(t)[\rho v]+[\rho u v+u]-\frac{d w(t) g(t)}{d t}\right\} \phi d t .
\end{aligned}
$$

Relation (79) is called the generalized Rankine-Hugoniot relation. It reflects the exact relationship among the limit states on two sides of the discontinuity, the weight, propagation speed, and the location of the discontinuity. In addition, to guarantee uniqueness, the delta shock wave should satisfy the admissibility (entropy) condition

$$
\lambda_{3}\left(\rho_{r}, u_{r}, v_{r}\right) \leq u_{\delta}(t) \leq \lambda_{1}\left(\rho_{l}, u_{l}, v_{l}\right) .
$$

Now, the generalized Rankine-Hugoniot relation is applied to the Riemann problem (2)-(44) with left and right constant states $U_{-}=\left(\rho_{-}, u_{-}, v_{-}\right)$and $U_{+}=\left(\rho_{+}, u_{+}, v_{+}\right)$, respectively, satisfying the conditions $(\mathrm{H} 1)$ and $(\mathrm{H} 2)$, the fact $\lambda_{3}\left(\rho_{+}, u_{+}, v_{+}\right) \leq \lambda_{1}\left(\rho_{-}, u_{-}, v_{-}\right)$, and

$$
\begin{aligned}
\frac{1}{2}\left(\lambda_{1}\left(U_{-}\right)-\lambda_{3}\left(U_{+}\right)\right)^{2} \\
\geq \max \left\{-\frac{s^{2}}{\rho_{+}}\left(R_{2}\left(U_{+}\right)-R_{2}\left(U_{-}\right)\right),\right. \\
\left.\frac{s^{2}}{\rho_{-}}\left(R_{2}\left(U_{+}\right)-R_{2}\left(U_{-}\right)\right)\right\} .
\end{aligned}
$$

Thereby, the Riemann problem is reduced to solving (79) with initial data

$$
t=0, \quad x(0)=0, \quad w(0)=0, \quad g(0)=0,
$$

under entropy condition

$$
u_{+}+\frac{s}{\rho_{+}} \leq u_{\delta}(t) \leq u_{-}-\frac{s}{\rho_{-}} .
$$

From (79) and (83), it follows that

$$
\begin{gathered}
w(t)=-[\rho] x(t)+[\rho u] t \\
w(t) u_{\delta}(t)=-[\rho u] x(t)+\left[\rho u^{2}+s^{2} v\right] t, \\
w(t) g(t)=-[\rho v] x(t)+[\rho u v+u] t .
\end{gathered}
$$

Multiplying the first equation in $(85)$ by $u_{\delta}(t)$ and then subtracting it from the second one, we obtain that

$$
[\rho] x(t) u_{\delta}(t)-[\rho u] u_{\delta}(t) t-[\rho u] x(t)+\left[\rho u^{2}+s^{2} v\right] t=0
$$

that is,

$$
\frac{d}{d t}\left(\frac{[\rho]}{2} x^{2}(t)-[\rho u] x(t) t+\frac{\left[\rho u^{2}+s^{2} v\right]}{2} t^{2}\right)=0,
$$


which is equivalent to

$$
[\rho] x^{2}(t)-2[\rho u] x(t) t+\left[\rho u^{2}+s^{2} v\right] t^{2}=0 .
$$

From (88), one can find that $u_{\delta}(t):=u_{\delta}$ is a constant and $x(t)=u_{\delta} t$. Then, (88) can be rewritten

$$
[\rho] u_{\delta}^{2}-2[\rho u] u_{\delta}+\left[\rho u^{2}+s^{2} v\right]=0 .
$$

When $[\rho]=\rho_{-}-\rho_{+}=0$, the situation is very simple and one can easily calculate the solution

$$
\begin{gathered}
u_{\delta}=\frac{u_{-}+u_{+}}{2}+s^{2} \frac{[v]}{2 \rho_{-}[u]}, \\
x(t)=u_{\delta} t, \\
w(t)=\rho_{-}\left(u_{-}-u_{+}\right) t, \\
g(t)=\frac{[\rho u v+u]-u_{\delta}}{[\rho u]},
\end{gathered}
$$

which obviously satisfies the entropy condition (84), since by condition (82),

$$
\begin{aligned}
s^{2} \frac{[v]}{\rho_{-}} & \leq \frac{1}{2}\left(\lambda_{1}\left(U_{-}\right)-\lambda_{3}\left(U_{+}\right)\right)^{2} \\
& <\frac{1}{2}[u]\left(\lambda_{1}\left(U_{-}\right)-\lambda_{3}\left(U_{+}\right)\right), \\
& u_{\delta}-\left(u_{-}-\frac{s}{\rho_{-}}\right) \\
& =\frac{u_{-}+u_{+}}{2}+s^{2} \frac{[v]}{2 \rho_{-}[u]}-\left(u_{-}-\frac{s}{\rho_{-}}\right) \\
& =\frac{1}{2}\left(\left(u_{+}+\frac{s}{\rho_{-}}\right)-\left(u_{-}-\frac{s}{\rho_{-}}\right)+s^{2} \frac{[v]}{\rho_{-}[u]}\right) \leq 0 .
\end{aligned}
$$

Similarly we can deduce that

$$
\begin{aligned}
& u_{\delta}-\left(u_{+}+\frac{s}{\rho_{-}}\right) \\
& =\frac{u_{-}+u_{+}}{2}+s^{2} \frac{[v]}{2 \rho_{-}[u]}-\left(u_{+}+\frac{s}{\rho_{-}}\right) \\
& =\frac{1}{2}\left(\left(u_{-}-\frac{s}{\rho_{-}}\right)-\left(u_{+}+\frac{s}{\rho_{-}}\right)+s^{2} \frac{[v]}{\rho_{-}[u]}\right) \geq 0,
\end{aligned}
$$

because

$$
\begin{aligned}
-s^{2} \frac{[v]}{\rho_{-}} & \leq \frac{1}{2}\left(\lambda_{1}\left(U_{-}\right)-\lambda_{3}\left(U_{+}\right)\right)^{2} \\
& <\frac{1}{2}[u]\left(\lambda_{1}\left(U_{-}\right)-\lambda_{3}\left(U_{+}\right)\right) .
\end{aligned}
$$

When $[\rho]=\rho_{-}-\rho_{+} \neq 0$, the discriminant of the quadratic equation (89) is

$$
\Delta=4[\rho u]^{2}-4[\rho]\left[\rho u^{2}+s^{2} v\right]=\rho_{-} \rho_{+}[u]^{2}-s^{2}[\rho][v]>0,
$$

and then we can find

$$
\begin{gathered}
u_{\delta}=\frac{[\rho u]-\sqrt{[\rho u]^{2}-[\rho]\left[\rho u^{2}+s^{2} v\right]}}{[\rho]}, \\
x(t)=\frac{[\rho u]-\sqrt{[\rho u]^{2}-[\rho]\left[\rho u^{2}+s^{2} v\right]}}{[\rho]} t, \\
w(t)=\sqrt{[\rho u]^{2}-[\rho]\left[\rho u^{2}+s^{2} v\right]} t, \\
g(t)=\left(\left(-[\rho u][\rho v]+[\rho v] \sqrt{[\rho u]^{2}-[\rho]\left[\rho u^{2}+s^{2} v\right]}\right.\right. \\
+[\rho][\rho u v+u]) \\
\left.\times\left([\rho] \sqrt{[\rho u]^{2}-[\rho]\left[\rho u^{2}+s^{2} v\right]}\right)^{-1}\right) t,
\end{gathered}
$$

or

$$
\begin{gathered}
u_{\delta}=\frac{[\rho u]+\sqrt{[\rho u]^{2}-[\rho]\left[\rho u^{2}+s^{2} v\right]}}{[\rho]}, \\
x(t)=\frac{[\rho u]+\sqrt{[\rho u]^{2}-[\rho]\left[\rho u^{2}+s^{2} v\right]}}{[\rho]} t, \\
w(t)=-\sqrt{[\rho u]^{2}-[\rho]\left[\rho u^{2}+s^{2} v\right]} t, \\
g(t)=\left(\left(-[\rho u][\rho v]-[\rho v] \sqrt{[\rho u]^{2}-[\rho]\left[\rho u^{2}+s^{2} v\right]}\right.\right. \\
+[\rho][\rho u v+u]) \\
\left.\times\left([\rho] \sqrt{[\rho u]^{2}-[\rho]\left[\rho u^{2}+s^{2} v\right]}\right)^{-1}\right) t .
\end{gathered}
$$

Next, with the help of the entropy condition (84), we will choose the admissible solution from (95) and (96). Observe that, by the entropy condition and since the system is strictly hyperbolic, we have that

$$
u_{+}-\frac{s}{\rho_{+}}<u_{+}<u_{+}+\frac{s}{\rho_{+}} \leq u_{-}-\frac{s}{\rho_{-}}<u_{-}<u_{-}+\frac{s}{\rho_{-}} .
$$

Observe that

$$
\begin{aligned}
& -[\rho] \lambda_{1}\left(\rho_{-}, u_{-}, v_{-}\right)+[\rho u] \\
& =\rho_{+}\left(\left(u_{-}-\frac{s}{\rho_{-}}\right)-\left(u_{+}-\frac{s}{\rho_{+}}\right)\right)>0 ; \\
& -[\rho] \lambda_{3}\left(\rho_{+}, u_{+}, v_{+}\right)+[\rho u] \\
& =\rho_{-}\left(\left(u_{-}+\frac{s}{\rho_{-}}\right)-\left(u_{+}+\frac{s}{\rho_{+}}\right)\right)>0,
\end{aligned}
$$




$$
\begin{aligned}
{[\rho]\left(\lambda_{1}\left(\rho_{-}, u_{-}, v_{-}\right)\right)^{2} } & \\
& -2[\rho u] \lambda_{1}\left(\rho_{-}, u_{-}, v_{-}\right)+\left[\rho u^{2}\right]+s^{2}[v] \\
= & -\rho_{+}\left(u_{-}-u_{+}-\frac{s}{\rho_{-}}\right)^{2}+\frac{s^{2}}{\rho_{-}}+s^{2}[v] \leq 0, \\
{[\rho]\left(\lambda_{3}\left(\rho_{+}, u_{+}, v_{+}\right)\right)^{2} } & -2[\rho u] \lambda_{3}\left(\rho_{+}, u_{+}, v_{+}\right)+\left[\rho u^{2}\right]+s^{2}[v] \\
& \quad \rho_{-}\left(u_{-}-u_{+}-\frac{s}{\rho_{+}}\right)^{2}-\frac{s^{2}}{\rho_{+}}+s^{2}[v] \geq 0,
\end{aligned}
$$

then for the solution given in (95), we have

$$
\begin{aligned}
& u_{\delta}-\lambda_{1}\left(\rho_{-}, u_{-}, v_{-}\right) \\
& =\left(\left([\rho]\left(\lambda_{1}\left(\rho_{-}, u_{-}, v_{-}\right)\right)^{2}-2[\rho u] \lambda_{1}\left(\rho_{-}, u_{-}, v_{-}\right)\right.\right. \\
& \left.+\left[\rho u^{2}\right]+s^{2}[v]\right) \\
& \times\left(\left(-[\rho] \lambda_{1}\left(\rho_{-}, u_{-}, v_{-}\right)+[\rho u]\right)\right. \\
& \left.\left.+\sqrt{[\rho u]^{2}-[\rho]\left[\rho u^{2}+s^{2} v\right]}\right)^{-1}\right) \leq 0, \\
& u_{\delta}-\lambda_{3}\left(\rho_{+}, u_{+}, v_{+}\right) \\
& =\left(\left([\rho]\left(\lambda_{3}\left(\rho_{+}, u_{+}, v_{+}\right)\right)^{2}-2[\rho u] \lambda_{3}\left(\rho_{+}, u_{+}, v_{+}\right)\right.\right. \\
& \left.+\left[\rho u^{2}\right]+s^{2}[v]\right) \\
& \times\left(\left(-[\rho] \lambda_{3}\left(\rho_{+}, u_{+}, v_{+}\right)+[\rho u]\right)\right. \\
& \left.\left.+\sqrt{[\rho u]^{2}-[\rho]\left[\rho u^{2}+s^{2} v\right]}\right)^{-1}\right) \geq 0,
\end{aligned}
$$

which imply that the entropy condition (84) is valid. When $\lambda_{1}\left(\rho_{-}, u_{-}, v_{-}\right)=\lambda_{3}\left(\rho_{+}, u_{+}, v_{+}\right)$, we have trivially that $\lambda_{1}\left(\rho_{-}, u_{-}, v_{-}\right)=u_{\delta}=\lambda_{3}\left(\rho_{+}, u_{+}, v_{+}\right)$.

Now, for the solution (96), when $\rho_{-}<\rho_{+}$we have

$$
\begin{aligned}
u_{\delta} & -\lambda_{3}\left(\rho_{+}, u_{+}, v_{+}\right) \\
& =\frac{-[\rho] \lambda_{3}\left(U_{+}\right)+[\rho u]+\sqrt{[\rho u]^{2}-[\rho]\left[\rho u^{2}+s^{2} v\right]}}{[\rho]} \\
& =\frac{\rho_{-}\left(\lambda_{3}\left(U_{-}\right)-\lambda_{3}\left(U_{+}\right)\right)+\sqrt{[\rho u]^{2}-[\rho]\left[\rho u^{2}+s^{2} v\right]}}{[\rho]} \\
& <0,
\end{aligned}
$$

and when $\rho_{-}>\rho_{+}$, that

$$
\begin{aligned}
u_{\delta} & -\lambda_{1}\left(\rho_{-}, u_{-}, v_{-}\right) \\
& =\frac{-[\rho] \lambda_{1}\left(U_{-}\right)+[\rho u]+\sqrt{[\rho u]^{2}-[\rho]\left[\rho u^{2}+s^{2} v\right]}}{[\rho]} \\
& =\frac{\rho_{+}\left(\lambda_{1}\left(U_{-}\right)-\lambda_{1}\left(U_{+}\right)\right)+\sqrt{[\rho u]^{2}-[\rho]\left[\rho u^{2}+s^{2} v\right]}}{[\rho]} \\
& >0,
\end{aligned}
$$

showing that the solution (96) does not satisfy the entropy condition (84).

Thus we have proved the following result.

Theorem 10. Given left and right constant states $\left(\rho_{l}, u_{l}, v_{l}\right)$ and $\left(\rho_{r}, u_{r}, v_{r}\right)$, respectively, such that satisfy the conditions $(H 1)$, (H2), $\lambda_{1}\left(\rho_{l}, u_{l}, v_{l}\right) \geq \lambda_{3}\left(\rho_{r}, u_{r}, v_{r}\right)$ and (82), that is,

$$
\begin{aligned}
& \frac{1}{2}\left(\lambda_{1}\left(U_{l}\right)-\lambda_{3}\left(U_{r}\right)\right)^{2} \\
& \geq \max \left\{-\frac{s^{2}}{\rho_{r}}\left(R_{2}\left(U_{r}\right)-R_{2}\left(U_{l}\right)\right), \frac{s^{2}}{\rho_{l}}\left(R_{2}\left(U_{r}\right)-R_{2}\left(U_{l}\right)\right)\right\} .
\end{aligned}
$$

Then, the Riemann problem (2)-(44) admits a unique entropy solution in the sense of measures. This solution is of the form

$$
(\rho, u, v)(t, x)= \begin{cases}\left(\rho_{l}, u_{l}, v_{l}\right), & \text { if } x<u_{\delta} t \\ \left(w(t) \delta\left(x-u_{\delta} t\right), u_{\delta}, g(t)\right), & \text { if } x=u_{\delta} t \\ \left(\rho_{r}, u_{r}, v_{r}\right), & \text { if } x>u_{\delta} t\end{cases}
$$

where $u_{\delta}, w(t)$, and $g(t)$ are shown in (90) for $[\rho]=0$ or $(95)$ for $[\rho] \neq 0$.

The above result includes the cases

$$
\frac{1}{s}\left(\lambda_{3}\left(U_{r}\right)-\lambda_{1}\left(U_{l}\right)\right)-\left(R_{2}\left(U_{r}\right)-R_{2}\left(U_{l}\right)\right)=0,
$$

or

$$
\frac{1}{s}\left(\lambda_{3}\left(U_{r}\right)-\lambda_{1}\left(U_{l}\right)\right)+\left(R_{2}\left(U_{r}\right)-R_{2}\left(U_{l}\right)\right)=0,
$$

that according to our knowledge is the best case of the delta shock waves (see (68)).

We began by analyzing the case in (104). Assume left and right constant states $\left(\rho_{l}, u_{l}, v_{l}\right)$ and $\left(\rho_{r}, u_{r}, v_{r}\right)$, respectively, such that satisfy the conditions $(\mathrm{H} 1)$ and $(\mathrm{H} 2), \lambda_{1}\left(\rho_{l}, u_{l}, v_{l}\right) \geq$ $\lambda_{3}\left(\rho_{r}, u_{r}, v_{r}\right)$, and (104).

It is easy to see that if $\lambda_{1}\left(\rho_{l}, u_{l}, v_{l}\right)=\lambda_{3}\left(\rho_{r}, u_{r}, v_{r}\right)$, then the inequality (82) is trivially satisfied. Suppose that $\lambda_{1}\left(\rho_{l}, u_{l}, v_{l}\right)>\lambda_{3}\left(\rho_{r}, u_{r}, v_{r}\right)$. Then, $\lambda_{3}\left(\rho_{r}, u_{r}, v_{r}\right)<$ $\lambda_{3}\left(\rho_{l}, u_{l}, v_{l}\right)$, and so

$$
\lambda_{3}\left(\rho_{r}, u_{r}, v_{r}\right)-\lambda_{1}\left(\rho_{l}, u_{l}, v_{l}\right)<\frac{2 s}{\rho_{l}},
$$

meaning that (82) is satisfied. The analysis is similar for (105). 


\section{Conflict of Interests}

The authors declare that there is no conflict of interests regarding the publication of this paper.

\section{Acknowledgments}

The authors are grateful to Professor Yunguang Lu for suggesting this problem, pointing out the simplification of the system proposed by Bouchut and Boyaval, and also communicating to us the paper [2]. Also, They are grateful to Professor Philippe G. LeFloch for bringing to our attention the references $[4,21,22]$.

\section{References}

[1] F. Bouchut and S. Boyaval, "A new model for shallow viscoelastic fluids," Mathematical Models and Methods in Applied Sciences, vol. 23, no. 8, pp. 1479-1526, 2013.

[2] Y.-G. Lu, C. Klingenberg, L. Rendon, and D.-Y. Zheng, "Global solutions for a simplified shallow elastic fluids model," Abstract and Applied Analysis, vol. 2014, Article ID 920248, 5 pages, 2014.

[3] F. Bouchut, Nonlinear Stability of Finite Volume Methods for Hyperbolic Conservation Laws and Well-Balanced Schemes for Sources, Frontiers in Mathematics, Birkhäuser, Basel, Switzerland, 2004.

[4] G. Carbou, B. Hanouzet, and R. Natalini, "Semilinear behavior for totally linearly degenerate hyperbolic systems with relaxation," Journal of Differential Equations, vol. 246, no. 1, pp. 291319, 2009.

[5] I. Suliciu, "On modelling phase transitions by means of ratetype constitutive equations. Shock wave structure," International Journal of Engineering Science, vol. 28, no. 8, pp. 829-841, 1990.

[6] P. Baiti and A. Bressan, "The semigroup generated by a Temple class system with large data," Differential and Integral Equations, vol. 10, no. 3, pp. 401-418, 1997.

[7] S. Bianchini, "The semigroup generated by a Temple class system with non-convex flux function," Differential and Integral Equations, vol. 13, no. 10-12, pp. 1529-1550, 2000.

[8] S. Bianchini, "Stability of $L^{\infty}$ solutions for hyperbolic systems with coinciding shocks and rarefactions," SIAM Journal on Mathematical Analysis, vol. 33, no. 4, pp. 959-981, 2001.

[9] A. Bressan, Hyperbolic Systems of Conservation Laws: The OneDimensional Cauchy Problem, vol. 20 of Oxford Lecture Series in Mathematics and Its Applications, Oxford University Press, Oxford, UK, 2000.

[10] A. Bressan and P. Goatin, "Stability of $L^{\infty}$ solutions of Temple class systems," Differential and Integral Equations, vol. 13, no. 1012, pp. 1503-1528, 2000.

[11] A. Heibig, "Existence and uniqueness of solutions for some hyperbolic systems of conservation laws," Archive for Rational Mechanics and Analysis, vol. 126, no. 1, pp. 79-101, 1994.

[12] Y.-J. Peng, "Euler-Lagrange change of variables in conservation laws," Nonlinearity, vol. 20, no. 8, pp. 1927-1953, 2007.

[13] D. Serre, "Solutions à variations bornées pour certains systèmes hyperboliques de lois de conservation," Journal of Differential Equations, vol. 68, no. 2, pp. 137-168, 1987.

[14] C. Chalons and F. Coquel, "Navier-Stokes equations with several independent pressure laws and explicit predictor-corrector schemes," Numerische Mathematik, vol. 101, no. 3, pp. 451-478, 2005.

[15] V. G. Danilov and V. M. Shelkovich, "Delta-shock wave type solution of hyperbolic systems of conservation laws," Quarterly of Applied Mathematics, vol. 63, no. 3, pp. 401-427, 2005.

[16] J. Q. Li and T. Zhang, "Generalized Rankine-Hugoniot relations of deltashocks in solutions of transportation equations," in Advance in Nonlinear PDE and Related Areas, pp. 219-232, World Scientific, Singapore, 1998.

[17] D. Serre, "Richness and the classification of quasilinear hyperbolic systems," IMA Preprint Series 597, 1989.

[18] D. H. Wagner, "Equivalence of the Euler and Lagrangian equations of gas dynamics for weak solutions," Journal of Differential Equations, vol. 68, no. 1, pp. 118-136, 1987.

[19] E. Weinan and R. V. Kohn, "The initial value problem for measure-valued solutions of a canonical $2 \times 2$ system with linearly degenerate fields," Communications on Pure and Applied Mathematics, vol. 44, no. 8-9, pp. 981-1000, 1991.

[20] T.-T. Li, Y.-J. Peng, and J. Ruiz, "Entropy solutions for linearly degenerate hyperbolic systems of rich type," Journal de Mathématiques Pures et Appliquées, vol. 91, no. 6, pp. 553-568, 2009.

[21] C. Chalons and J.-F. Coulombel, "Relaxation approximation of the Euler equations," Journal of Mathematical Analysis and Applications, vol. 348, no. 2, pp. 872-893, 2008.

[22] F. Coquel, E. Godlewski, and N. Seguin, "Relaxation of fluid systems," Mathematical Models and Methods in Applied Sciences, vol. 22 , no. 8, 52 pages, 2012 . 


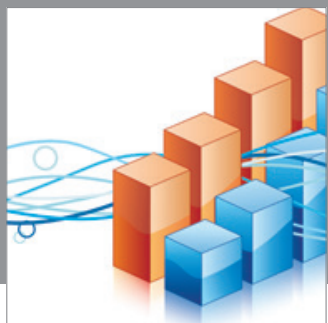

Advances in

Operations Research

mansans

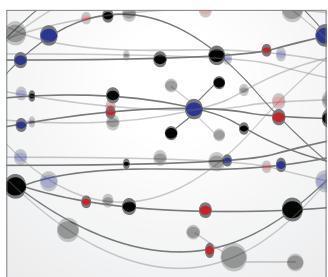

The Scientific World Journal
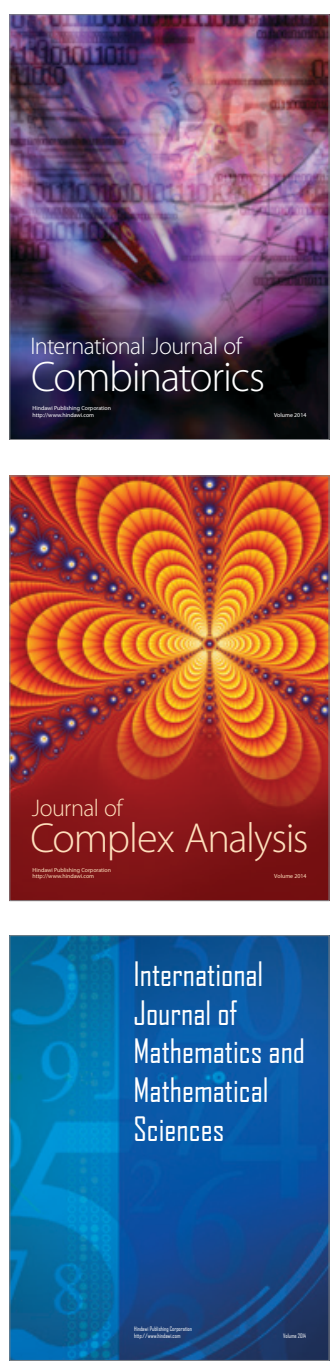
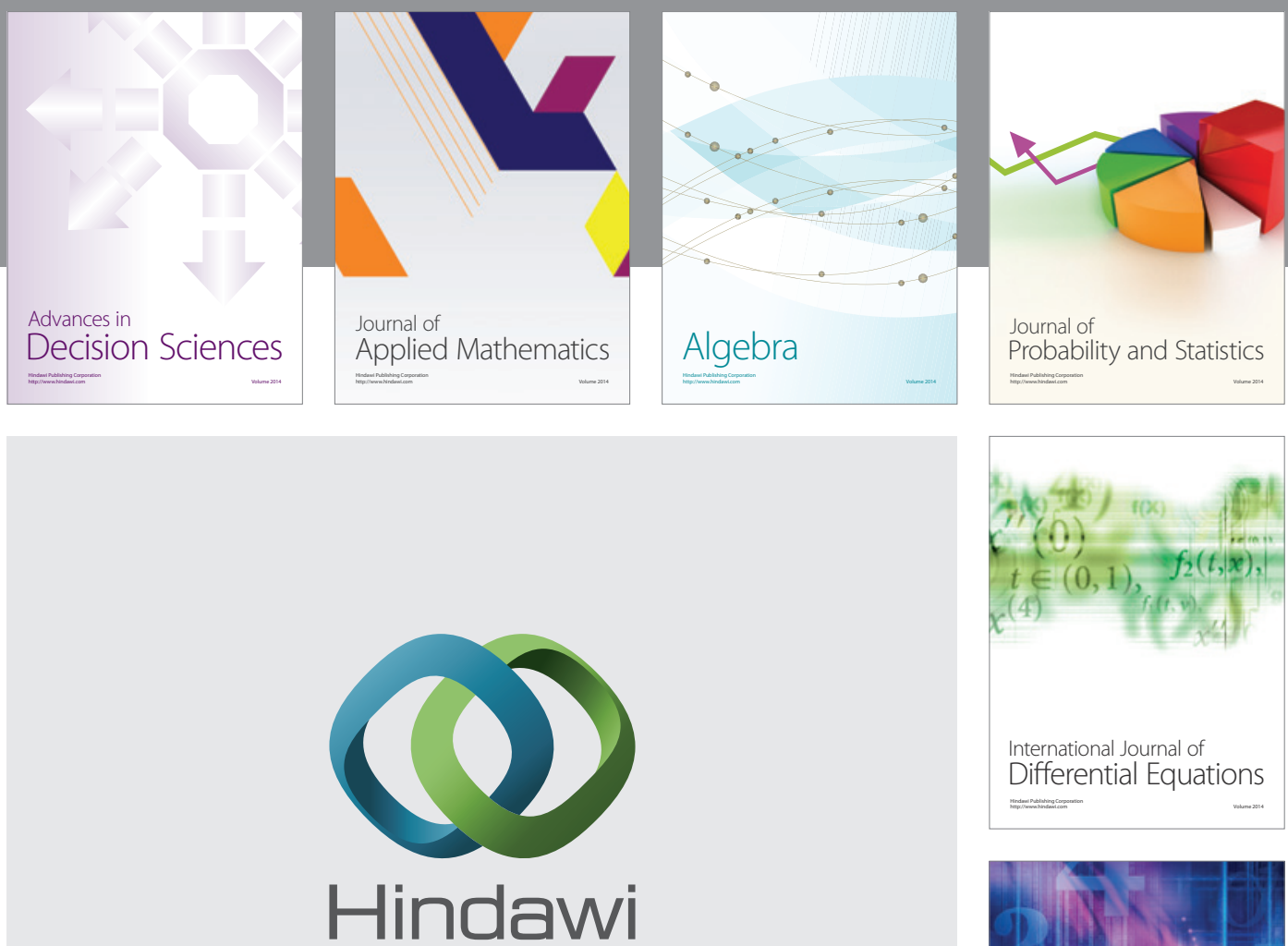

Submit your manuscripts at http://www.hindawi.com
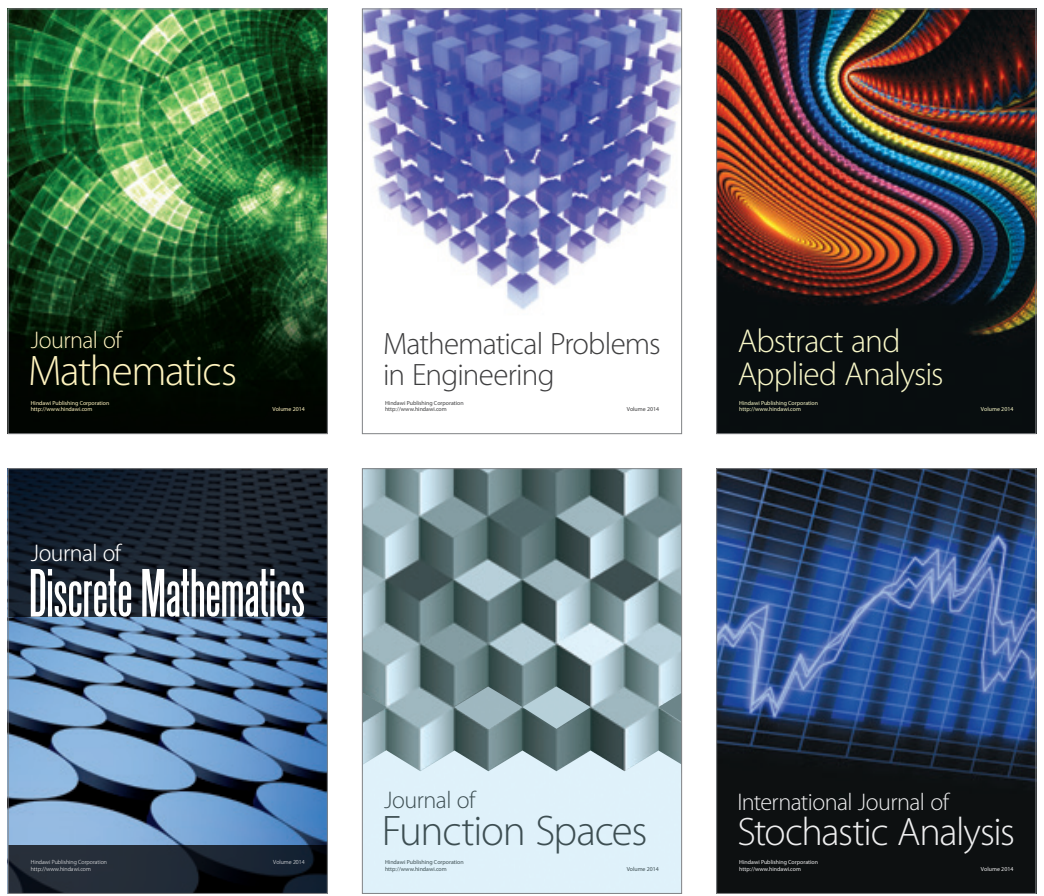

Journal of

Function Spaces

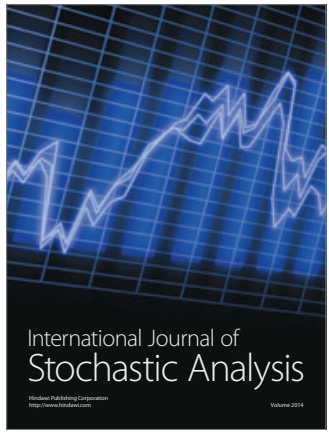

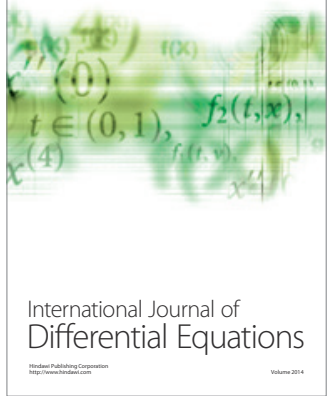
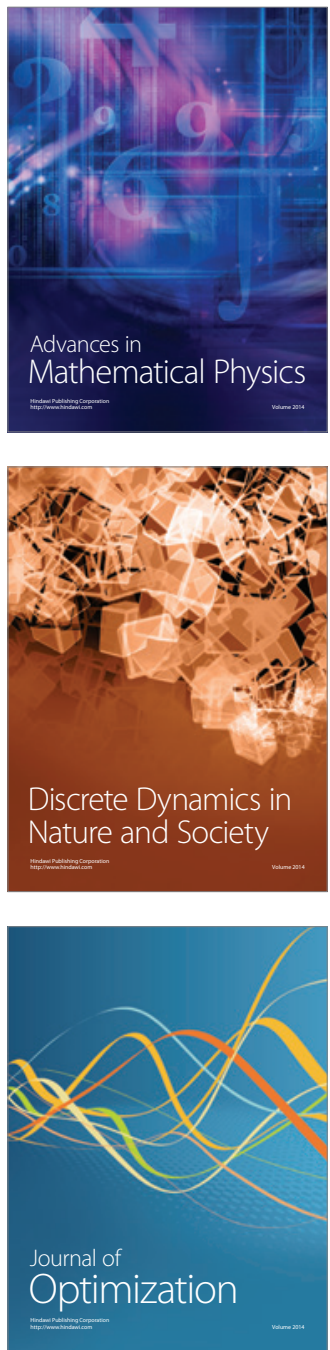